\title{
A study on the Sensitivity of Parameterizations for Regional Climate models in the Simulation of Tropical Cyclones over Western Pacific Ocean and East Sea
}

\author{
Pham Quang Nam ${ }^{1}$, Tran Quang Duc ${ }^{1, *}$, Le Lan Phuong ${ }^{2}$, Hoang Danh Huy ${ }^{1}$, \\ Pham Thanh $\mathrm{Ha}^{1}$, Phan Van Tan ${ }^{1}$ \\ ${ }^{1}$ VNU University of Science, 334 Nguyen Trai, Hanoi, Vietnam \\ ${ }^{2}$ National Central University, 300 Zhongda Road, Taoyuan, Taiwan
}

Received 20 February 2020

Revised 02 May 2020; Accepted 07 May 2020

\begin{abstract}
This study investigates the sensitivity of physical parameterization schemes in two regional dynamic models clWRF (the climate Weather Research and Forecasting) and RegCM (the Regional Climate Model) in the simulation of tropical cyclones (TCs) over Western Pacific Ocean and East Sea. The experiments include 12-cases for clWRF model and 6-cases for RegCM model were conducted to run the simulation, with the same domain parameters, resolution $25 \mathrm{~km}$. Results show that the clWRF can simulate TCs well with the Betts-Miller-Janjic convection scheme and WSM6 microphysics, in which convection schemes are more influential, and the RegCM is with the Kain-Fritsch convection scheme and Zeng oceanic flux. Regarding the number of TCs simulation, most of them are higher than observed and CFSnl (Climate Forecast System analysis) data, therein the RegCM is higher than the clWRF.
\end{abstract}

Keywords: parameterizations, regional models, tropical cyclones, Western Pacific Ocean, East Sea.

\footnotetext{
* Corresponding author.

E-mail address: tranquangduc@hus.edu.vn

https://doi.org/10.25073/2588-1094/vnuees.4568
} 


\title{
Nghiên cứu độ nhạy của sơ đồ tham số hóa vật lý trong mô hình khí hậu khu vực đối với mô phỏng hoạt động của xoáy thuận nhiệt đới trên Tây Bắc Thái Bình Dương và Biển Đông
}

\author{
Phạm Quang Nam ${ }^{1}$, Trần Quang Đức ${ }^{1, *}$, Lê Lan Phương ${ }^{2}$, Hoàng Danh Huy ${ }^{1}$, \\ Phạm Thanh Hà ${ }^{1}$, Phan Văn Tân ${ }^{1}$ \\ ${ }^{I}$ Truò̀ng Đại học Khoa học Tụ nhiên, Đại học Quốc gia Hà Nội, 334 Nguyễn Trãi, Hà Nội, Việt Nam \\ ${ }^{2}$ Truờng Đại học Quốc gia Trung uoong, 300 Trung Đại, Trung Lịch, Đào Viên, Đài Loan \\ Nhận ngày 20 tháng 02 năm 2020 \\ Chỉnh sửa ngày 02 tháng 5 năm 2020; Chấp nhận đăng ngày 07 tháng 5 năm 2020
}

\begin{abstract}
Tóm tắt: Nghiên cứu này khảo độ nhạy của các sơ đồ tham số hóa vật lý trong hai mô hình động lực khu vực clWRF (the climate Weather Research and Forecast) và RegCM (the Regional Climate Model) cho việc mô phỏng xoáy thuận nhiệt đới (XTNĐ) trên khu vực Tây Bắc Thái Bình Dương và Biển Đông. Với 12 thí nghiệm cho mô hình clWRF và 6 thí nghiệm cho mô hình RegCM đã được tiến hành chạy mô phỏng, với cùng các thông số về miền tính, phân giải $25 \mathrm{~km}$. Kết quả chỉ ra mô hình clWRF cho mô phỏng XTNĐ tốt với sơ đồ tham số hóa đối lưu Betts-Miller-Janjic và vi vật lý WSM6, trong đó sơ đồ đối lưu có ảnh hưởng nhiều hơn, còn đối với mô hình RegCM thì là với sơ đồ đối lưu Kain-Fritsch và thông lượng đại dương Zeng. Về số lượng XTNĐ mô phỏng, hầu hết cho cao hơn quan trắc và số liệu phân tích CFSnl (Climate Forecast System analysis), trong đó mô hình RegCM cho cao hơn clWRF.
\end{abstract}

Tù khoá: tham số hóa, mô hình khu vực, xoáy thuận nhiệt đới, Tây Bắc Thái Bình Dương, Biển Đông.

\section{Mở đầu}

Việt Nam với đường bờ biển dài trên 3000 $\mathrm{km}$, chạy dọc theo hướng Bắc-Nam, hàng năm phải chịu ảnh hưởng của trung bình khoảng 11 xoáy thuận nhiệt đới (XTNĐ) trên Biển Đông $\mathrm{BD})$, với thời gian hoạt động là từ tháng 6 đến tháng 11 (Ngữ và Hiệu, 2013) [1]. Sự xuất hiện của XTNĐ thường kèm theo các hiện tượng thời tiết nguy hiểm như gió giật, mưa lớn,... và gây ra những thiệt hại lớn đối với nền kinh tế-xã hội ở những nơi mà nó đi qua. Với các hoạt động kinh tế-xã hội diễn ra nhiều ngày, thậm chí hàng tháng, trên biển, như đánh bắt hải sản, tuần tra, tìm kiếm cứu nạn,... thì hạn dự báo của các bản

\footnotetext{
*Tác giả liên hệ.

Địa chỉemail: tranquangduc@hus.edu.vn

https://doi.org/10.25073/2588-1094/vnuees.4568
}

tin thời tiết không còn khả năng đáp ứng. Do đó, việc dự báo được số lượng, thời gian và quỹ đạo XTNĐ hoạt động trước từ một vài tháng, hoặc trước khi mùa bão bắt đầu, mang một ý nghĩa vô cùng quan trọng và thiết thực.

Thập kỷ gần đây, việc sử dụng các mô hình khí hậu toàn cầu (GCM) để dự báo sự hoạt động của xoáy thuận nhiệt đới (XTNĐ) hạn mùa đã được thực hiện khá thành công (LaRow và ccs, 2010; Zhao và ccs, 2010; Chen và Lin, 2011, 2013; Murakami và ccs, 2016) [2-6]. Không giống như các phương pháp thống kê truyền thống, là đi tìm mối quan hệ tuyến tính trong quá khứ rồi đem áp dụng nó cho các dự báo ở hiện tại, với phương pháp động lực sử dụng mô hình 
toàn cầu, các XTNĐ có cường độ và kích thước khác nhau có thể xuất hiện một cách tự nhiên ở những nơi mà có môi trường quy mô lớn thuận lợi cho việc hình thành (Chen và Lin, 2013) [5]. Mặc dù có thể sử dụng trực tiếp các thông tin dự báo toàn cầu cho quy mô khu vực, tuy nhiên hoặc độ phân giải GCM vẫn còn thô (phổ biển khoảng $100 \mathrm{~km}$ ) hoặc chạy GCM với phân giải cao nhưng sẽ tốn rất nhiều tài nguyên máy tính không thực tế khi đưa vào nghiệp vụ. Vì vậy, các mô hình khí hậu khu vực (RCM) sẽ được sử dụng để làm chi tiết hóa các dự báo từ GCM (Zhan và ccs, 2012; Warner, 2011) [7, 8], và nó như là một bước trung gian cần thực hiện trước khi tiến hành các bước tiếp theo để dự báo XTNĐ.

Ngày nay, với sự tiến bộ của khoa học kỹ thuật, năng lực tính toán của hệ thống máy tính được nâng lên rất nhiều, việc chạy các RCM cho bài toán dự báo thời tiết thông thường có thể được thực hiện với phân giải ngang cao cỡ nhỏ hơn $10 \mathrm{~km}$, nên một số quá trình vật lý có thể được tính toán ngay trên lưới thay vì phải tham số hóa bằng các sơ đồ. Tuy nhiên, đối với bài toán dự báo mùa nói chung và dự báo XTNĐ hạn mùa nói riêng, thời gian mô hình chạy trên hệ thống tính toán là tương đối dài, cỡ vài ngày hoặc hàng tuần, và đòi hỏi không gian lưu trữ lớn, như vậy việc chạy các RCM với phân giải cao giống như dự báo thời tiết là không khả thi, dẫn đến phân giải được coi là cao cỡ khoảng $15-25 \mathrm{~km}$. Do đó, việc đánh giá độ nhạy của các sơ đồ tham số hóa vật lý mô hình tới kết quả dự báo hoặc mô phỏng XTNĐ là rất quan trọng trước khi đem áp dụng mô hình đó cho nghiệp vụ.

Về độ nhạy của các vật lý mô hình, một số nghiên cứu đã phân tích ảnh hưởng của tham số đối lưu đối với sự hình thành XTNĐ (Smith, 2000; Sanderson và ccs, 2008; Zhao và ccs, 2012) [9-11]. Các nghiên cứu khác đánh giá độ nhạy của số lượng XTNĐ toàn cầu đối với các tham số đối lưu (v.d. LaRow và ccs, 2008; Ma và Tan, 2009; Reed và Jablonowski, 2011) [1214]. Cụ thể, Ma và Tan (2009) [13], đã so sánh hiệu suất của ba sơ đồ đối lưu và thây rằng sơ đồ Kain-Fritsch $(\mathrm{KF})$ phát triển bởi Kain và Fritsch (1993) [15] cho mô phỏng tốt nhất XTNĐ với phân giải lưới $15 \mathrm{~km}$, trong khi sơ đồ bán-cân bằng đề xuất bởi Grell (1993) [16] lại có xu hướng ước tính thấp lượng mưa quy mô dưới lưới. Diro và ccs (2014) [17] cũng đã cho thấy sơ đồ Grell cho ước tính thấp sản phẩm XTNĐ ở Bắc Đại Tây Dương và Đông Thái Bình Dương, trong khi sơ đồ Emanuel (1991) [18] cho hiệu suất tốt hơn đáng kể. Trong nghiên cứu của Sun và ccs (2014) [19], cơn bão Megi được mô phỏng lại bằng Mô hình Nghiên cứu và Dự báo thời tiết - WRF (the Weather Research and Forecast) chạy với các sơ đồ tham số đối lưu GrellDevenyi (GD) và Betts-Miller-Janjic (BMJ), kết quả cho thấy quỹ đạo bão có thể được tái tạo tốt với sơ đồ $\mathrm{GD}$, trong khi cơn bão chuyển hướng sớm hơn với sơ đồ $\mathrm{BMJ}$. Kanase và Salvekar (2015) [20] lại cho thấy rằng quỹ đạo và cường độ của cơn bão Laila mô phỏng bằng mô hình WRF đã cho tốt với sơ đồ đối lưu $\mathrm{BMJ}$, và rất nhạy với mô tả môi trường quy mô lớn trong sơ đồ đối lưu, còn với sơ đồ vi vật lý WSM6 thì có thể tác động đến cường độ cơn bão. Một khía cạnh khác của vật lý mô hình cũng có tầm quan trọng đặc biệt đối với mô phỏng XTNĐ là sự trao đổi năng lượng, độ ẩm, và động lượng giữa khí quyển và đại dương (Zeng và ccs, 2010) [21], như nghiên cứu của Fuentes-Franco và ccs (2016) [22], hiệu suất mô phỏng XTNĐ trên vùng nhiệt đới phía đông Thái Bình Dương và nhiệt đới Bắc Đại Tây Dương, của mô hình RegCM (the Regional Climate Model) tốt nhất đạt được khi sử dụng kết hợp sơ đồ đối lưu KF và sơ đồ thông lượng đại dương Zeng (Zeng và ccs, 1998) [23], với phân giải lưới $25 \mathrm{~km}$.

Ở Việt Nam, bài toán mô phỏng và dự báo XTNĐ hạn mùa thì chưa có nhiều nghiêu cứu chuyên sâu và việc ứng dụng đưa vào dự báo nghiệp vụ vẫn còn nhiều hạn chế. Trong số ít đó, phải kể đến nghiên cứu bước đầu có đóng góp quan trọng của Hải và Tân (2010) [24], các tác giả sử dụng mô hình khu vực RegCM3, phân giải $54 \mathrm{~km}$, chạy với số liệu tái phân tích ERA40 và nhiệt độ bề mặt biển OISST để mô phỏng sự hoạt động của XTNĐ trên khu vực Tây Bắc Thái Bình Dương (TBTBD) và $\mathrm{BĐ}$, kết quả thử nghiệm cho thấy đã mô phỏng được sự hoạt động của XTNĐ, trong đó một sản phẩm quan trọng là nhóm tác giả đã xây dựng được bộ chương trình dò tìm 
XTNĐ từ sản phẩm mô hình, các xoáy bão tìm được gọi là xoáy tựa bão hay xoáy tựa xoáy thuận nhiệt đới (Tropical cyclone like vortices TCLV). Một nghiên cứu tiếp theo cũng của nhóm tác giả (Phan và ccs, 2015) [25] nhưng là về dự báo XTNĐ, các tác giả sử dụng mô hình RegCM phiên bản mới hơn 4.2, phân giải $36 \mathrm{~km}$, để chi tiết hóa dự báo từ mô hình toàn cầu $\mathrm{CFS}$ (Climate Forecasting System), sản phẩm mô hình được tiến hành dò tìm xoáy bão bằng thuật toán dò tìm đã được cải tiến giúp phát hiện các trung tâm xoáy hiệu quả hơn, kết quả cho thấy RegCM dự báo một cách hợp lý phân bố số lượng XTNĐ theo thời gian cũng như quỹ đạo XTNĐ.

Như vậy, qua trích dẫn bên trên, có thể thấy rằng vấn đề nghiên cứu độ nhạy của tham số vật lý mô hình đối với các tính chất của XTNĐ đã chưa thực sự được bắt đầu ở Việt Nam. Do đó, nghiên cứu này sẽ là thử nghiệm bước đầu về độ nhạy của một số các sơ đồ tham số thường được sử dụng trong các mô hình khu vực đối với việc mô phỏng số lượng và quỹ đạo XTNĐ trên TBTBD và $B Đ$. Chi tiết về các mô hình động lực được sử dụng, cấu hình miền tính, các sơ đồ tham số, cùng với các ngưỡng chỉ tiêu dò tìm XTNĐ sẽ được trình bày ở phần 2 , kết quả và thảo luận ở phần 3 .

\section{Phương pháp, số liệu, và thiết kế thí nghiệm}

\subsection{Các mô hình động lục}

Mô hình WRF, phiên bản 3.9.1, phi thủy tĩnh, gồm hệ các phương trình nguyên thủy ba chiều (Skamarock và ccs, 2008) [26], với phần mở rộng phục vụ cho mô phỏng khí hậu, viết tắt là clWRF (the climate WRF), được sử dụng trong nghiên cứu này. Mô hình clWRF được bổ sung các mô-đun cho phép sử dụng với các kịch bản phát thải khí nhà kính, và tính toán các biến cực trị phục vụ cho bài toán nghiên cứu khí hậu và biến đổi khí hậu. Nhằm đánh giá độ nhạy của các sơ đồ tham số hóa đối lưu và vi vật lý của mô hình clWRF trong việc mô phỏng XTNĐ, nghiên cứu sẽ chạy mô hình với 12 thí nghiệm, gồm 4 sơ đồ đối lưu và 3 sơ đồ vi vật lý khác nhau (bảng $1)$. Vì có rất nhiều các tùy chọn sơ đồ tham số hóa cho mô hình clWRF, nên không thể tiến hành tất các thí nghiệm, nên ở đây chỉ sử dụng các sơ đồ đã được đánh giá trong các nghiên cứu về độ nhạy mà cho mô phỏng tốt XTNĐ (v.d. Sun và ccs, 2014, 2015; Kanase và Salvekar, 2015; Islam và ccs, 2015; Chan và ccs, 2016) $[19,27,20,28,29]$. Ngoài các sơ đồ đã thiết lập cho mỗi thí nghiệm, các sơ đồ khác được sử dụng chung bao gồm sơ đồ: bức xạ sóng ngắn, sóng dài RRTMG, bề mặt đất Noah LSM, và lớp biên hành tinh YSM (http://www2.mmm.ucar.edu/ wrf/users/docs/user_guide_V3/ARWUsersGuid eV3.9.pdf, lần truy cập cuối: 20/01/2020). Mô hình clWRF được cấu hình để chạy với phân giải ngang $25 \mathrm{~km}, 36$ mực thẳng đứng, thời gian tích phân là 60 giây, tâm miền tính nằm ở $13,6^{\circ} \mathrm{B}$ và $125^{\circ} \mathrm{Ð}$, và nằm trong khoảng $0-27^{\circ} \mathrm{B}$ và $90-160^{\circ}$ , miền tính này đã được xem xét và thiết lập một cách tối ưu, sau khi khảo sát kỹ phần địa hình (vì có ảnh hưởng lớn, đặc biệt trong trường hợp biên miền tính nằm trên các khu vực có địa hình phức tạp, ví dụ cao nguyên Tây Tạng), và khu vực XTNĐ hoạt động trên $\mathrm{BĐ}$, cũng như trên khu vực TBTBD.

Một mô hình nữa cũng được sử dụng trong nghiên cứu này là $\mathrm{RegCM}$, phiên bản 4.7 , với các lỗi đã được khắc phục (Giorgi và ccs, 2016) [30], và tăng cường hiệu suất mô hình. RegCM4.7 là thủy tĩnh, nhưng nó cũng bao gồm cả một lõi phi thủy tĩnh, nén được, mô hình khu vực giới hạn với hệ tọa độ lai sigma-pressure (Nellie và ccs, 2014) [31]. Cũng nhằm đánh giá độ nhạy của các sơ đồ tham số trong việc mô phỏng và dự báo XTNĐ của mô hình RegCM, nghiên cứu tiến hành 6 thí nghiệm với mô hình $\mathrm{RegCM}$, bao gồm 3 sơ đồ tham số đối lưu bề mặt và đại dương, và 2 sơ đồ thông lượng đại dương (bảng 2), các sơ đồ này cũng đã được sử dụng trong các nghiên cứu như của Fuentes-Franco và ccs (2016) [22], Lok và Chan (2018) [32], Vishnu và ccs (2019) [33]. Ở đây, RegCM được chạy với cấu hình thủy tĩnh, sơ đồ đất CLM (the Community Land Model) phiên bản 4.5, phân giải ngang mô hình là $25 \mathrm{~km}, 18$ mực thẳng đứng, thời gian tích phân là 30 giây, về thông số miền tính thì cũng được thiết lập giống như cho mô hình clWRF. 
Bảng 1. Tên các thí nghiệm được thực hiện với mô hình clWRF

\begin{tabular}{|c|c|c|c|}
\hline TT & Tên thí nghiệm & Tham số hóa đối lưu & Tham số hóa vi vật lý \\
\hline 1 & clWRF_exp1 & Kain-Fritsch & WSM6 \\
\hline 2 & clWRF_exp2 & New Tiedtke & WSM6 \\
\hline 3 & clWRF_exp3 & Grell-Freitas & WSM6 \\
\hline 4 & clWRF_exp4 & Betts-Miller-Janjic & WSM6 \\
\hline 5 & clWRF_exp5 & Kain-Fritsch & Thompson \\
\hline 6 & clWRF_exp6 & New Tiedtke & Thompson \\
\hline 7 & clWRF_exp7 & Grell-Freitas & Thompson \\
\hline 8 & clWRF_exp8 & Betts-Miller-Janjic & Thompson \\
\hline 9 & clWRF_exp9 & Kain-Fritsch & WDM6 \\
\hline 10 & clWRF_exp10 & New Tiedtke & WDM6 \\
\hline 11 & clWRF_exp11 & Grell-Freitas & WDM6 \\
\hline 12 & clWRF_exp12 & Betts-Miller-Janjic & WDM6 \\
\hline
\end{tabular}

Bảng 2. Tên các thí nghiệm được thực hiện với mô hình RegCM

\begin{tabular}{|c|c|c|c|}
\hline TT & Tên thí nghiệm & $\begin{array}{c}\text { Tham số hóa đối lưu } \\
\text { bề mặt và đại dương }\end{array}$ & $\begin{array}{c}\text { Tham số hóa thông } \\
\text { lượng đại dương }\end{array}$ \\
\hline 1 & RegCM_exp1 & Emanuel & BATS1e \\
\hline 2 & RegCM_exp2 & Tiedtke & BATS1e \\
\hline 3 & RegCM_exp3 & Kain-Fritsch & BATS1e \\
\hline 4 & RegCM_exp4 & Emanuel & Zeng \\
\hline 5 & RegCM_exp5 & Tiedtke & Zeng \\
\hline 6 & RegCM_exp6 & Kain-Fritsch & Zeng \\
\hline
\end{tabular}

\subsection{Nguồn số liệu}

Số liệu tái phân tích CFSR (Climate Forecast System Reanalysis) hoặc phân tích CFSnl (CFS analysis) với độ phân giải $0,5 \times 0,5$ độ kinh vĩ, 37 mực thẳng đứng, được sử dụng làm điều kiện ban đầu và điều kiện biên xung quanh cho các mô hình khu vực. CFSR được xây dựng cho 31 năm, giai đoạn 1979-2009 và được mở rộng như là một hoạt động nghiệp vụ tới nay với tên gọi là số liệu phân tích CFSnl. Những điểm nổi bật của CFSnl bao gồm: có sự kết hợp giữa khí quyểnđại dương trong quá trình tạo ra trường phỏng đoán 6 giờ, tương tác với mô hình băng biển, và đồng hóa số liệu bức xạ vệ tinh bằng hệ thống đồng hóa GSI (Gridpoint Statistical Interpolation) (Saha và ccs, 2010) [34].

Quỹ đạo và số lượng bão hoạt động trên trên khu vực TBTBD được sử dụng trong việc đánh giá sự mô phỏng của các mô hình là số liệu quan trắc của JTWC (the Joint Typhoon Warning Center). JTWC có thể được tải về từ trang web: https://www.metoc.navy.mil/jtwc/jtwc.html?we stern-pacific.

\subsection{Thiết kế thi nghiệm}

Nghiên cứu này sẽ chạy các mô hình khu vực mô phỏng XTNĐ cho các năm 2010 và 2013, trong đó năm 2010 là năm có ít bão, và 2013 là năm nhiều bão hoạt động. Thời điểm chạy mô hình là $00 \mathrm{Z}$ ngày 01 tháng 12 của năm trước đó (2009 và 2012), chạy như vậy là để cho mô hình có một khoảng thời gian khởi động (spin-up). Kết quả đầu ra của mô hình sẽ được xử lý và tiến hành dò tìm XTNĐ bằng bộ chương trình TCs detect (Hải và Tân, 2010; Phan và ccs, 2015) $[23,24]$. Tuy nhiên, vì mỗi mô hình là một hệ động lực khác nhau nên cần phải có các ngưỡng chỉ tiêu riêng áp dụng phù hợp cho việc chạy TCs_detect, do đó nghiên cứu sẽ tiến hành dò tìm XTNĐ với khoảng 42 bộ ngưỡng chỉ tiêu cho mỗi trường hợp mô phỏng của mô hình, cũng như đối với số liệu CFSnl. Tiêu chí để xem xét 
trường hợp nào cho mô phỏng XTNĐ tốt nhất là dựa vào số lượng và quỹ đạo so với số liệu CFSnl và quan trắc.

Các ngưỡng chỉ tiêu cho việc dò tìm XTNĐ đối với số liệu CFSnl, và mô phỏng của các mô hình được lựa chọn như trong bảng 3. Cần lưu ý rằng ngưỡng chỉ tiêu phụ thuộc vào độ phân giải của số liệu, với phân giải cao thì giá trị tuyệt đối của ngưỡng sẽ lớn hơn so với phân giải thấp. Do việc dò tìm tâm XTNĐ được thực hiện tại mỗi lát thời gian cách nhau 6 giờ, nên trong quá trình dò tìm XTNĐ cần quan tâm thêm đến việc tâm XTNĐ dò tìm được là ở thời điểm này hay là của XTNĐ đã tồn tại trước đó, xác định bằng cách kiểm tra xem sự tồn tại của XTNĐ tại lát thời gian trước có nằm trong vòng bán kính $300 \mathrm{~km}$ tính từ vị trí tâm XTNĐ được tìm thấy tại thời điểm này không. Để loại bỏ những nhiễu động trong quá trình dò xoáy, chỉ những xoáy tồn tại ít nhất 2 ngày (48 giờ, 8 ốp) thì mới được coi là các XTNĐ.

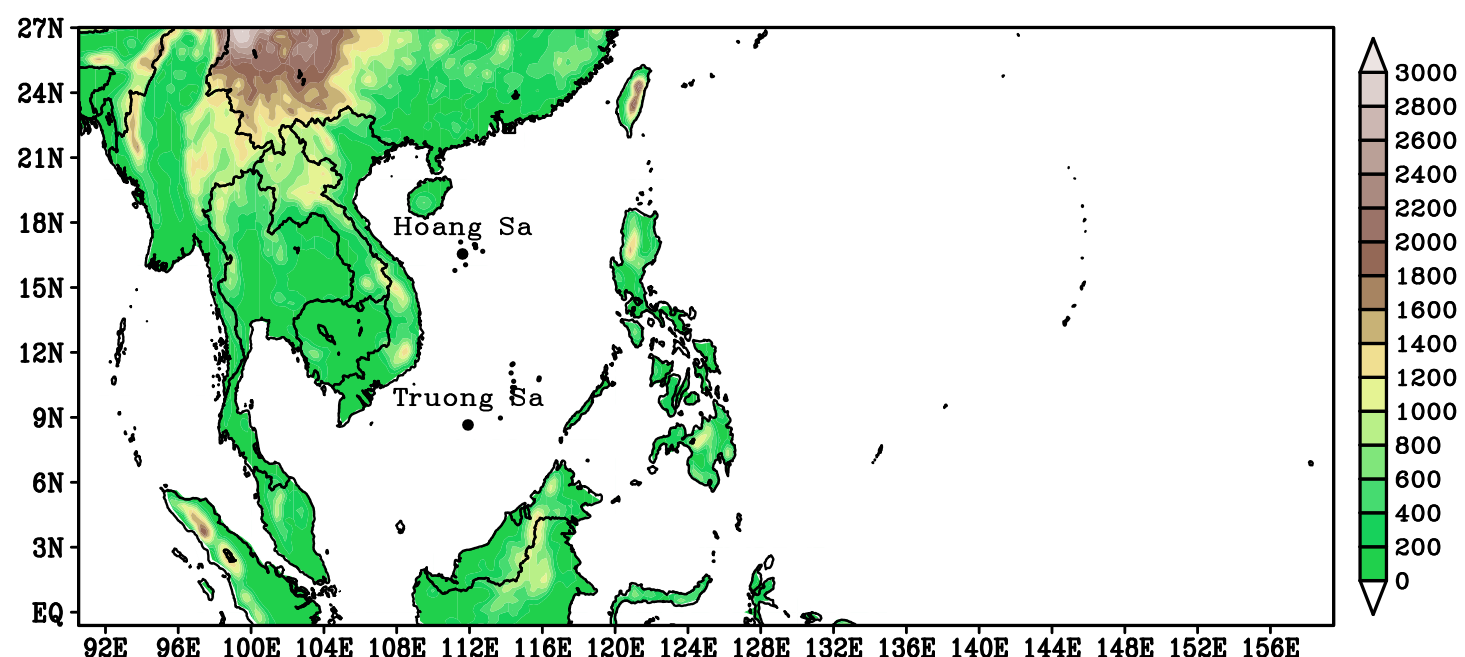

Hình 1. Minh họa miền tính và độ cao địa hình $(\mathrm{m})$ của mô hình clWRF.

Bảng 3. Các ngưỡng chỉ tiêu dò tìm XTNĐ áp dụng cho số liệu CFSnl và mô phỏng của các mô hình

\begin{tabular}{|c|c|c|c|c|}
\hline & Độ xoáy $\left(\mathrm{s}^{-1}\right)$ & Dị thường lõi nóng $(K)$ & Tốc độ gió OCS (m/s) & Dị thường áp suất (hPa) \\
\hline CFSnl & $5 \times 10^{-5}$ & 0,2 & 4,5 & -2 \\
\hline clWRF & $5 \times 10^{-5}$ & 1 & 5 & -5 \\
\hline RegCM & $5 \times 10^{-5}$ & 1 & 5 & -3 \\
\hline
\end{tabular}

\section{Kết quả và thảo luận}

Trên bảng 4 là tổng số XTNĐ hoạt động trong mùa bão (từ tháng 6 đến tháng 11) các năm 2010 và 2013 , của số liệu quan trắc, và số lượng dò tìm được với ngưỡng chỉ tiêu được cho là tốt nhất của số liệu CFSnl và các trường hợp mô phỏng (thí nghiệm từ 1 đến 12 ). Kết quả cho thấy, số liệu CFSnl dò tìm được số lượng XTNĐ hoạt động bằng với quan trắc, trong khi các trường hợp mô phỏng có sự biến động đáng kể. Đối với các trường hợp mô phỏng của clWRF, sự ảnh hưởng của tham số hóa đối lưu đối với kết quả mô phỏng mạnh hơn so với tham số vi vật lý, nguyên nhân dễ hiểu là do sự trao đổi đối lưu trong XTNĐ diễn ra liên tục nên chỉ cần sai khác nhỏ trong mô tả của sơ đồ cũng gây ra ảnh hưởng lớn. Điều này được minh chứng ở số lượng 
XTNĐ, v.d. các trường hợp clWRF_exp1, clWRF exp5, và clWRF_exp9 với cùng một sơ đồ đối lưu Kain-Fritsch thì đều cho nhiều XTNĐ, trong khi các trường hợp clWRF_exp2, clWRF_exp6, và clWRF_exp10 với sơ đồ đối lưu New Tiedtke thì cho ít XTNĐ. Các trường hợp mô phỏng clWRF_exp4, clWRF_exp7, và clWRF_exp12 có số lượng XTNĐ trong hai năm tiệm cận gần hơn so với quan trắc nên sẽ được sử dụng để đánh giá chi tiết ở phần quỹ đạo hoạt động XTNĐ tiếp sau đây. Còn đối với các trường hợp mô phỏng của RegCM thì có thể dễ dàng nhận ra trường hợp RegCM exp6 với sơ đồ Kain-Fritsch và Zeng đã cho mô phỏng tốt nhất.

Bảng 4. Tổng số lượng XTNĐ từ tháng 6 đến tháng 11 của các năm 2010 và 2013 , của quan trắc, số liệu CFSnl, và của các trường hợp mô phỏng

\begin{tabular}{|c|c|c|c|c|c|c|c|c|c|c|c|c|c|c|c|}
\hline \multirow{2}{*}{ Năm } & \multirow{2}{*}{ Mô hình } & \multicolumn{12}{|c|}{ Thí nghiệm } & \multirow[b]{2}{*}{ Obs } & \multirow{2}{*}{ CFSnl } \\
\hline & & 1 & 2 & 3 & 4 & 5 & 6 & 7 & 8 & 9 & 10 & 11 & 12 & & \\
\hline \multirow{2}{*}{2010} & clWRF & 37 & 6 & 29 & 17 & 27 & 5 & 23 & 24 & 38 & 5 & 39 & 20 & \multirow{2}{*}{16} & \multirow{2}{*}{16} \\
\hline & RegCM & 6 & 6 & 39 & 2 & 0 & 19 & & & & & & & & \\
\hline \multirow{2}{*}{2013} & clWRF & 43 & 16 & 44 & 38 & 40 & 14 & 40 & 34 & 45 & 15 & 49 & 33 & \multirow{2}{*}{31} & \multirow{2}{*}{31} \\
\hline & RegCM & 16 & 11 & 48 & 4 & 2 & 37 & & & & & & & & \\
\hline
\end{tabular}

Quỹ đạo XTNĐ hoạt động của số liệu CFSnl trong năm 2013 nhìn chung khá tương đồng so với quan trắc về đường đi và thời điểm bắt đầu hình thành bão, ví dụ như cơn bão Haiyan bắt đầu từ ngày $3 / 11$, vị trí hình thành khoảng $155^{\circ}$ $Đ$ và $6^{\circ} \mathrm{B}$ với quan trắc, còn với $C F S n l$ là khoảng $153^{\circ} \mathrm{Đ}$ và $6^{\circ} \mathrm{B}$ (Hình 2 , thứ tự 1 và 2 từ trên xuống). Đối với năm 2010 thì số liệu CFSnl có quỹ đạo bắt kém hơn, và chỉ được vài cơn là có quỹ đạo tựa giống bão quan trắc, v.d. cơn bão bắt đầu ngày $19 / 3$ của quan trắc và ngày $22 / 3$ của số liệu CFSnl. Các trường hợp mô phỏng XTNĐ của các mô hình thì nhìn chung là quỹ đạo còn bị "rối", và đường đi khá dày trong khoảng vĩ độ từ $16^{\circ} \mathrm{B}$ đến $24^{\circ} \mathrm{B}$. Với clWRF thì có thể nhận thấy là các trường clWRF_exp4 và clWRF_exp12 chạy với sơ đồ đối lưu Betts-Miller-Janjic đã cho quỹ đạo mô phỏng tốt hơn trường hợp clWRF_exp7, và XTNĐ tìm thấy thường tập trung ở khoảng vĩ độ từ $16^{\circ} \mathrm{B}$ đến $24^{\circ} \mathrm{B}$ trong cả hai năm 2010 và 2013. Với trường hợp RegCM_exp6 thì mặc dù các XTNĐ được tìm thấy vẫn còn khá sát nhau về phân bố không gian, nhưng nhìn chung là có sự hợp lý hơn so với các mô phỏng của clWRF.

Để có thêm cơ sở khách quan trong việc đánh giá, nghiên cứu đã tiến hành tính toán giá trị khí áp mực biển cực tiểu theo thời gian cho các năm
2010 và 2013, của số liệu CFSnl, và các trường hợp mô phỏng, kết quả được thể hiện trên hình 3. Ở đây, có thể thấy rằng vùng hoạt động của XTNĐ sẽ tương ứng với vùng có giá trị khí áp thấp nhất trên bản đồ. Trường hợp clWRF_exp7 trong cả hai năm đều cho giá trị khí áp thấp hơn và phân tán hơn so với số liệu CFSnl, trong khi các trường hợp clWRF_exp4 và clWRF_exp12 thì có sự tương đồng và đều cho khí áp cao hơn CFSnl, nhưng có thể nhận thấy là trường hợp clWRF_exp4 đã cho mô phỏng tốt hơn cả, cụ thể: giá trị khí áp năm 2010 cao hơn và năm 2013 thấp hơn trường hợp clWRF_exp12, điều này hoàn toàn hợp lý vì năm 2010 là năm ít bão nên giá trị khí áp sẽ lớn hơn 2013. Với trường hợp RegCM_exp6, mô phỏng cũng đã thể hiện được phần nào vùng có XTNĐ hoạt động, về giá trị vùng khí áp cực tiểu thì vẫn cho cao hơn so với CFSnl.

Tiếp theo, số lượng bão hoạt động theo tháng của hai mùa bão năm 2010 và 2013 của quan trắc, và số lượng $X T N Đ$ của số liệu $C F S n l$, và của các trường hợp cho mô phỏng tốt nhất clWRF_exp4 và RegCM_exp6 được thể hiện trên hình 4 . Nhìn chung số lượng XTNĐ của các trường hợp mô phỏng thì đều cho cao hơn quan trắc và $\mathrm{CFSnl}$, trong đó mô hình $\mathrm{RegCM}$ cho cao hơn clWRF. 

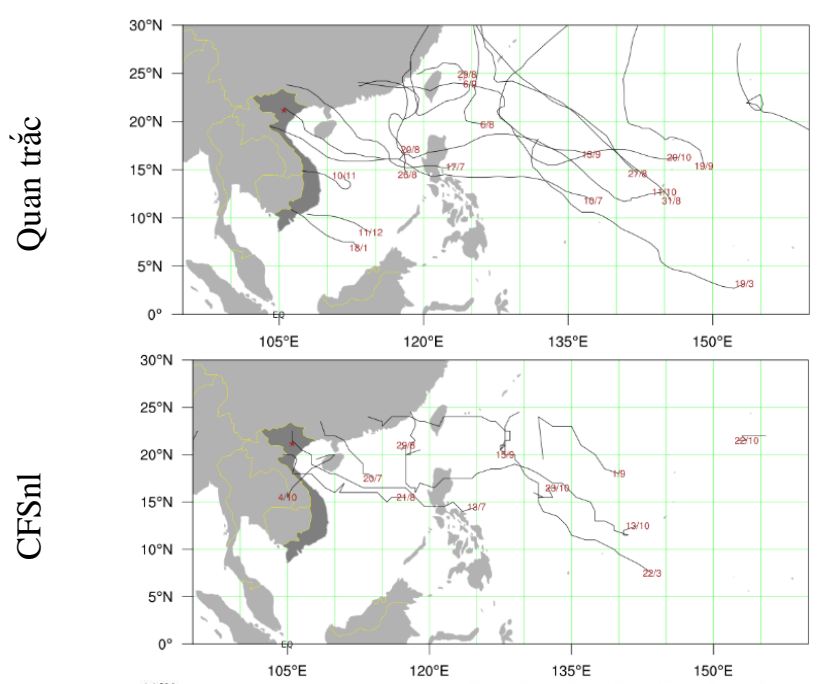

志

余

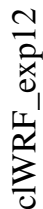

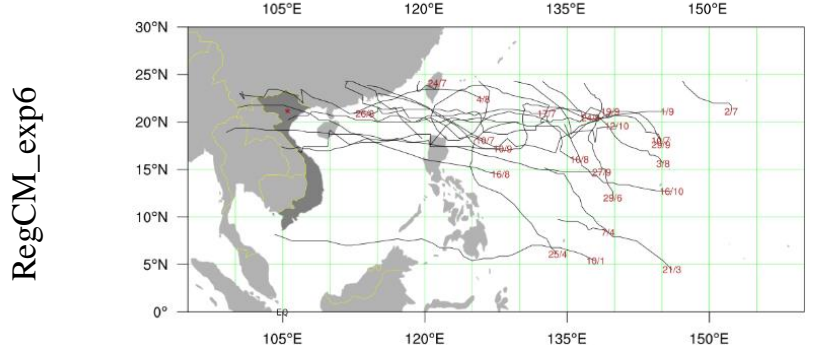

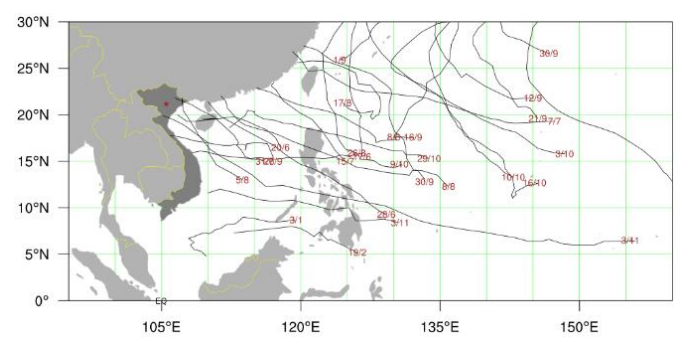
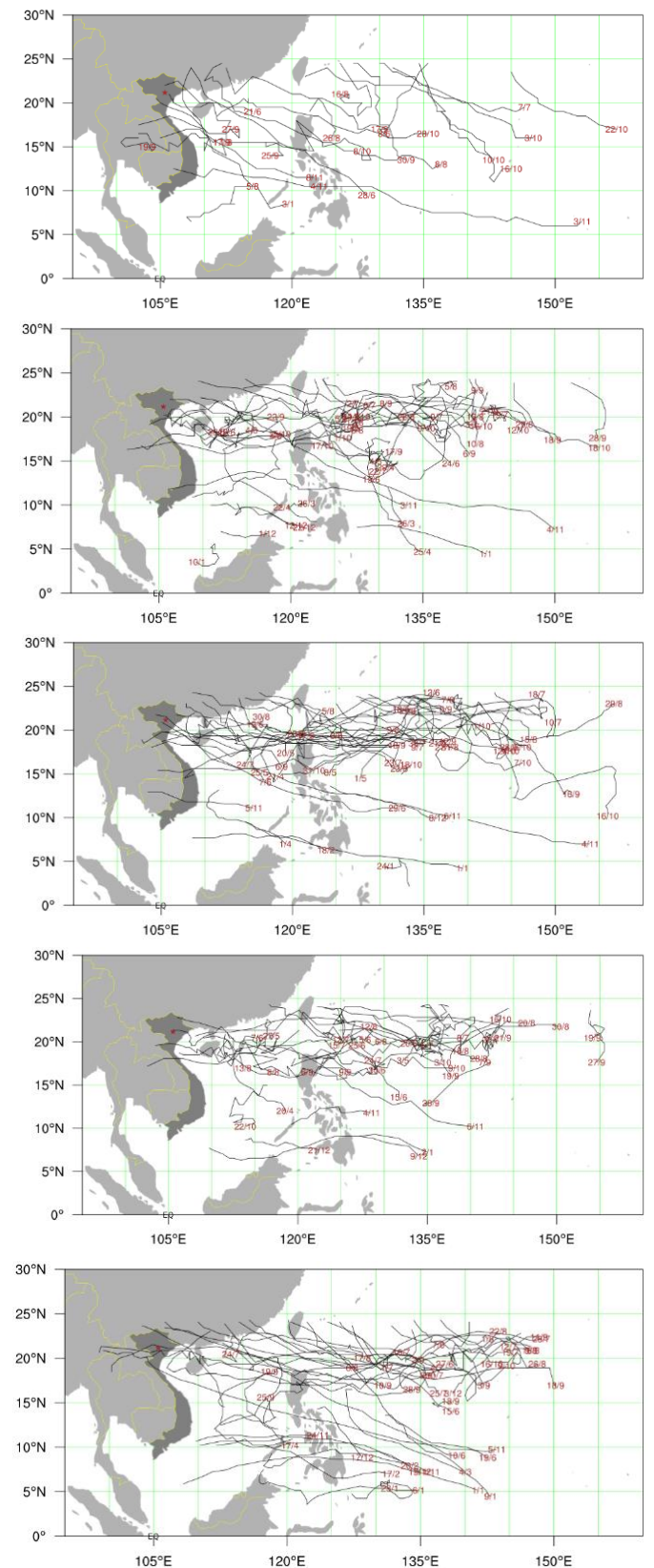

Hình 2. Quỹ đạo XTNĐ hoạt động trong các năm 2010 (bên trái) và 2013 (bên phải), của quan trắc, của số liệu CFSnl, và của các trường hợp mô phỏng. 

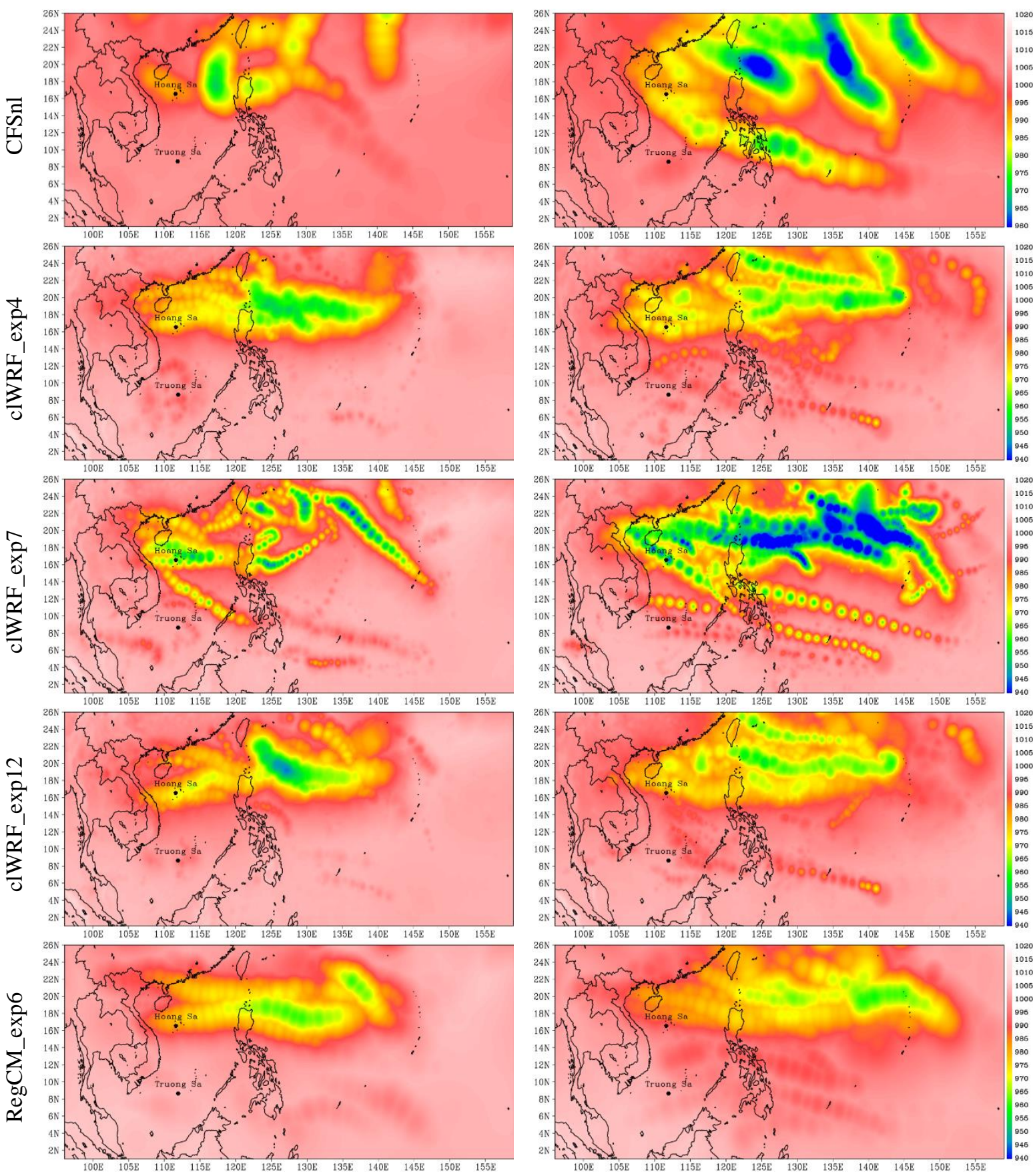

Hình 3. Khí áp cực tiểu theo thời gian của các năm 2010 (bên trái) và 2013 (bên phải), của số liệu CFSnl, và của các trường hợp mô phỏng. 

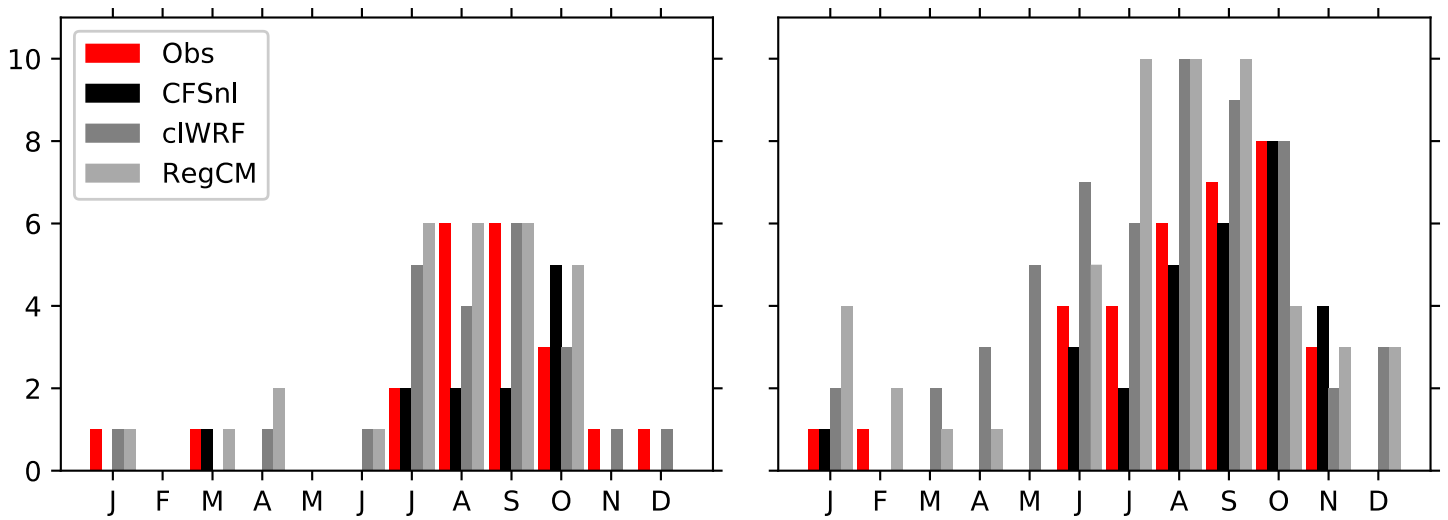

Hình 4. Biến trình số lượng XTNĐ trong các năm 2010 (bên trái) và 2013 (bên phải), của quan trắc, số liệu CFSnl, và của các trường hợp cho mô phỏng tốt nhất clWRF_exp4 và RegCM_exp6.

\section{Kết luận}

Với mục đích khảo sát về độ nhạy vật lý mô hình, nghiên cứu đã tiến hành chạy thử nghiệm hai mô hình khu vực clWRF và RegCM, với các sơ đồ tham số hóa khác nhau, phân giải $25 \mathrm{~km}$, để mô phỏng sự hoạt động của XTNĐ trên TBTBD và $B Đ$, cho các năm ít bão 2010 và nhiều bão 2013. Số liệu tái phân tích/phân tích CFS được sử dụng làm điều kiện ban đầu và điều kiện biên xung quanh cho các mô hình. XTNĐ của các trường hợp mô phỏng được dò tìm bằng bộ chương trình TCs_detect với các ngưỡng chỉ tiêu thích hợp. Qua việc phân tích đánh giá cho phép rút ra được các nhận xét sau đây:

i) Mô hình clWRF chạy với sơ đồ tham số hóa đối lưu Betts-Miller-Janjic và vi vật lý WSM6 đã cho mô phỏng tốt về số lượng và quỹ đạo XTNĐ hoạt động, trong đó sơ đồ đối lưu có ảnh hưởng nhiều hơn. Đối với mô hình $\mathrm{RegCM}$ thì là trường hợp kếp hợp của sơ đồ đối lưu KainFritsch và thông lượng đại dương Zeng, kết luận này cũng giống với nghiên cứu của FuentesFranco và ccs [22].

ii) Số lượng XTNĐ của các trường hợp mô phỏng hầu hết cho cao hơn quan trắc và CFSnl, trong đó mô hình RegCM cho cao hơn clWRF.

Những kết quả thu được về cấu hình, tham số hóa mô hình này sẽ là cắn cứ tham khảo quan trọng cho việc chạy các mô hình với đầu vào là số liệu dự báo CFS, để dự báo sự hoạt động của XTNĐ trong tương lai trước một vài tháng.

\section{Lời cảm ơn}

Nghiên cứu này được thực hiện và hoàn thành dưới sự hỗ trợ của Đề tài "Nghiên cứu xây dựng hệ thống dự báo số lượng và vùng hoạt động của bão trên Biển Đông hạn 3-6 tháng phục vụ hoạt động kinh tế biển và an ninh quốc phòng”, mã số: KC.09.15/16-20. Các tác giả xin chân thành cảm ơn.

\section{Tài liệu tham khảo}

[1] N.D. Ngu, N.T. Hieu, Climate and Climate Resources of Vietnam, Hanoi Science and Technology publisher, 2013.

[2] T.E. LaRow, L. Stefanova, D.W. Shin, S. Cocke, Seasonal Atlantic tropical cyclone hindcasting/ forecasting using two sea surface temperature datasets, Geophys. Res. Lett., 37 (2010). https:// doi.org/10.1029/2009GL041459.

[3] M. Zhao, I.M. Held, G.A. Vecchi, Retrospective forecasts of the hurricane season using a global atmospheric model assuming persistence of SST anomalies, Mon. Wea. Rev., 138 (2010) 38583868. https://doi.org/10.1175/2010MWR3366.1.

[4] J.H. Chen, S.J. Lin, The remarkable predictability of inter-annual variability of Atlantic hurricanes during the past decade, Geophys. Res. Lett., 38 (2011). https://doi.org/10.1029/2011GL047629. 
[5] J.H. Chen, S.J. Lin, Seasonal predictions of tropical cyclones using a 25-km-resolution general circulation model, J. Climate, 26 (2013) 380-398. https://doi.org/10.1175/JCLI-D-12-00061.1.

[6] H. Murakami, G.A. Vecchi, G. Villarini, T.L. Delworth, R. Gudgel, S. Underwood, X. Yang, W. Zhang, S. Lin, Seasonal forecasts of major hurricanes and landfalling tropical cyclones using a high-resolution GFDL coupled climate model, J. Climate, 29 (2016) 7977-7989. https://doi.org/10. 1175/JCLI-D-16-0233.1.

[7] R. Zhan, Y. Wang, and M. Ying, Seasonal forecasts of tropical cyclone activity over the western North Pacific: A review, Tropical Cyclone Research and Review, 1 (2012) 307-324.

[8] T.T. Warner, Numerical weather and climate prediction, Cambridge University Press, 2011.

[9] R.K. Smith, The role of cumulus convection in hurricanes and its representation in hurricane models, Rev, Geophys., 38 (2000) 465-489. https:// doi.org/10.1029/1999RG000080.

[10] B.M. Sanderson, C. Piani, W. Ingram, D. Stone, and M. Allen, Towards constraining climate sensitivity by linear analysis of feedback patterns in thousands of perturbed-physics GCM simulations, Clim. Dyn., 30 (2008) 175-190. https: //doi.org/10.1007/s00382-007-0280-7.

[11] M. Zhao, I.M. Held, S.J. Lin, Some counterintuitive dependencies of tropical cyclone frequency on parameters in a GCM. J. Atmos. Sci., 69 (2012) 2272-2283. https://doi.org/10.1175/JASD-11-0238.1.

[12] T. LaRow, Y.K. Lim, D. Shin, E. Chassignet, S. Cocke, Atlantic basin seasonal hurricane simulations, J. Clim., 21 (2008) 3191-3206. https://doi.org/10.11 75/2007JCLI2036.1.

[13] L.M. Ma, Z.M. Tan, Improving the behavior of the cumulus parameterization for tropical cyclone prediction: Convection trigger, Atmospheric Research, 92 (2009) 190-211. https://doi.org/10.10 16/j.atmosres.2008.09.022.

[14] K.A. Reed, C. Jablonowski, Impact of physical parameterizations on idealized tropical cyclones in the Community Atmosphere Model, Geophys Res Lett, 38 (2011). https://doi.org/10.1029/2010GL0 46297.

[15] J.S. Kain, J.M. Fritsch, Convective parameterization for mesoscale models: the Kain-Fritsch scheme. In: The representation of cumulus convection in numerical models, American Meteorological Society, (1993) 165-170.
[16] G. Grell, Prognostic evaluation of assumptions used by cumulus parameterizations, Mon. Wea. Rev., 121 (1993) 764-787.

[17] G.T. Diro, F. Giorgi, R. Fuentes-Franco, K.J.E. Walsh, G. Giuliani, E. Coppola, Tropical cyclones in a regional climate change projection with RegCM4 over the CORDEX Central America domain, Clim. Change, 125 (2014) 79-94.

[18] K. Emanuel, A scheme for representing cumulus convection in large scale models, J. Atmos. Sci., 48 (1991) 2313-2335.

[19] Y. Sun, Z. Zhong, W. Lu, Y. Hu, Why are tropical cyclone tracks over the Western North Pacific sensitive to the cumulus parameterization scheme in Regional Climate Modeling? A case study for Megi (2010), Mon. Wea. Rev., 142 (2014) 12401249.https://doi.org/10.1175/MWR-D-13-00232.1.

[20] R.D. Kanase, P.S. Salvekar, Effect of physical parameterization schemes on track and intensity of cyclone LAILA using WRF model, Asia-Pacific Journal of Atmospheric Sciences, 51 (2015) 205227.

[21] Z.H. Zeng, Y. Wang, Y.H. Duan, L.S. Chen, Z. Gao, On sea surface roughness parameterization and its effect on tropical cyclone structure and intensity, Adv. Atmos. Sci., 27 (2010) 337-355.

[22] R. Fuentes-Franco, F. Giorgi, E. Coppola, K. Zimmermann, Sensitivity of tropical cyclones to resolution, convection scheme and ocean flux parameterization over Eastern Tropical Pacific and Tropical North Atlantic Oceans in the RegCM4 model, Climate dynamics, 49 (2017) 547-561. https://doi.org/10.1007/s00382-016-3357-3.

[23] X. Zeng, M. Zhao, R.E. Dickinson, Intercomparison of bulk aerodynamic algorithms for the computation of sea surface fluxes using TOGA COARE and TAO data, J. Clim., 11 (1998) 26282644.

[24] B.H. Hai, P.V. Tan, Developing a tropical cyclonelike vortex scheme for RegCM model to simulate tropical cyclone activity over the West North Pacific and Vietnam East Sea, Journal of Hydrometeorology 8 (584) (2009) 1-8.

[25] V.T. Phan, T.T. Long, B.H. Hai, C. Kieu, Seasonal forecasting of tropical cyclone activity in the coastal region of Vietnam using RegCM4.2, Clim. Res., 62 (2015) 115-129. https://doi.org/10.3354/ cr01267.

[26] W.C. Skamarock, J.B. Klemp, J. Dudhia, D.O. Gill, D.M. Barker, M.G. Duda, X.Y. Huang, W. Wang, and J.G. Powers, A description of the advanced research WRF version 3, NCAR Technical Note NCAR/TN-475CSTR, (2008). 
[27] Y. Sun, Z. Zhong, H. Dong, J. Shi, Y. Hu, Sensitivity of tropical cyclone track simulation over the western North Pacific to different heating/drying rates in the Betts-Miller-Janjic scheme, Mon. Wea. Rev., 143 (2015) 3478-3494. https://doi.org/10.1175/MWR-D-14-00340.1.

[28] T. Islam, P.K. Srivastava, M.A. Rico-Ramirez, Q. Dai, M. Gupta, and S. K. Singh, Tracking a tropical cyclone through WRF-ARW simulation and sensitivity of model physics, Natural Hazards, 76 (2015) 1473-1495. https://doi.org/10.1007/s11069 $-014-1494-8$

[29] K.T. Chan, J.C. Chan, Sensitivity of the simulation of tropical cyclone size to microphysics schemes, Advances in Atmospheric Sciences, 33 (2016) 1024-1035. https://doi.org/10.1007/s00376-0165183-2.

[30] F. Giorgi, F. Solmon, G. Giuliani, Regional Climatic Model RegCM, User's Guide v4.6, ICTP, (2016).

[31] E. Nellie, B. Xunqiang, G. Filippo, N. Badrinath, P. Jeremy, S. Fabien, R. Sara, Z. Ashraf, O. Travis, N. Rita, and G. Graziano, Regional Climate Model RegCM reference manual v4.6, ICTP, (2014).

[32] C.C.F. Lok., J.C.L. Chan, Simulating seasonal tropical cyclone intensities at landfall along the
South China coast. Climate dynamics, 50 (2018) 2661-2672. https://doi.org/10.1007/s00382-0173762-2.

[33] S. Vishnu, J. Sanjay, and R. Krishnan, Assessment of climatological tropical cyclone activity over the north Indian Ocean in the CORDEX-South Asia regional climate models, Climate Dynamics, 53 (2019) 5101-5118. https://doi. org/10.1007/s00382 -019-04852-8.

[34] S. Saha, S. Moorthi, H. Pan, X. Wu, J. Wang, S. Nadiga, P. Tripp, R. Kistler, J. Woollen, D. Behringer, H. Liu, D. Stokes, R. Grumbine, G. Gayno, J. Wang, Y. Hou, H. Chuang, H.H. Juang, J. Sela, M. Iredell, R. Treadon, D. Kleist, P. Van Delst, D. Keyser, J. Derber, M. Ek, J. Meng, H. Wei, R. Yang, S. Lord, H. van den Dool, A. Kumar, W. Wang, C. Long, M. Chelliah, Y. Xue, B. Huang, J. Schemm, W. Ebisuzaki, R. Lin, P. Xie, M. Chen, S. Zhou, W. Higgins, C. Zou, Q. Liu, Y. Chen, Y. Han, L. Cucurull, R.W. Reynolds, G. Rutledge, M. Goldberg, The NCEP Climate Forecast System Reanalysis, Bull. Amer. Meteor. Soc., 91 (2010) 1015-1058. https://doi.org/10. 1175/2010BAMS3001.1. 